\title{
Taking steps to slow the upswing in oral and pharyngeal cancers
}

\author{
The CDC estimates that $70 \%$ of these cancers are caused \\ by HPV. And yet, in 2018, only about two-thirds of \\ adolescents had received 1 or more doses of HPV vaccine.
}

$\mathrm{A}$ recent report by the Centers for Disease Control and Prevention (CDC) documents the trends in oral and pharyngeal cancers (OPC) in the United States over a 10-year period, 2007-2016. ${ }^{1}$ The rate of OPC began to increase in 1999 and has been increasing ever since. The age-adjusted rate in 2007 was $10.89 / 100,000$ compared with $11.7 / 100,000$ in 2016 (TABLE 1 ${ }^{1}$ ). This is an annual relative increase of about $6 \%$ per year. In absolute numbers, there were 35,076 cases in 2007 and 44,419 in 2016. ${ }^{1}$ The trends in incidence of OPC vary by anatomical site, with some increasing and others declining.

There are 3 known causal factors related to OPC: tobacco use, alcohol use, and human papillomavirus (HPV) infection. The CDC estimates that, overall, $70 \%$ of OPCs are caused by $\mathrm{HPV}^{2}$ However, while cancers at some oropharyngeal sites are likely related to HPV infection, cancers at other sites are not. The rising overall incidence of OPC is being driven by increases in HPV-related cancers at an average rate of $2.1 \%$ per year, while the rates at non-HPV-associated sites have been declining by $0.4 \%$ per year. ${ }^{1}$ It is also important to appreciate that HPV causes cancer at other anatomical sites (TABLE $2^{2}$ ) and is responsible for an estimated 35,000 cancers per year. ${ }^{2}$

Other trends of note in all OPCs combined are increasing rates among nonHispanic whites and Asian-Pacific Islanders; decreasing rates among Hispanics and African Americans; increasing rates among males with no real change in rates among females; increasing rates in those 50 to 79 years of age; decreasing rates among those 40 to 49 years of age; and unchanged rates in other age groups. ${ }^{1}$

\section{The role of the family physician}

Preventing OPC and all HPV-related cancers begins by encouraging patients to reduce alcohol and tobacco use and by emphasizing the importance of HPV vaccination. Educate teens and parents/guardians about HPV vaccine and its safety. Screen for tobacco and alcohol use, and offer brief clinical interventions as needed to decrease usage.

Recommendations by the US Preventive Services Task Force regarding screening for, and reducing use of, tobacco and alcohol, as well as screening for cervical cancer, are listed in TABLE $3^{3.6}$ Remember that cervical cancer screening is both a primary and secondary intervention: It can reduce mortality by preventing cervical cancer (via treatment of precancerous lesions) and by detecting cervical cancer early at more treatable stages.

I HPV vaccination essentials. CDC recommendations for the use of HPV vaccine and the vaccine dosing schedule appear in TABLE $4 .{ }^{7}$ While it is true that the best evidence for HPV vaccine's prevention of cancer comes from the study of cervical and anal cancers, it is reasonable to expect that it will also be proven over time to prevent other HPV-caused cancers as the rate of HPV infections declines.

I HPV vaccine is underused. In a 2018 survey, only $68.1 \%$ of adolescents had received

\author{
Doug Campos-Outcalt, \\ MD, MPA \\ University of Arizona, \\ Phoenix \\ Ddougco@email.arizona. \\ edu
}


There is some evidence that if clinicians actively engage with parents about their vaccination concerns and address them head on, same-day vaccination rates can improve.

\section{TABLE 1}

\section{Number and rate of HPV-associated oral and pharyngeal} cancers by anatomic site, 2007 and $2016^{1}$

\begin{tabular}{l|l|l}
\hline Anatomic site & No. of cases $\left(\right.$ and rate $\left.{ }^{a}\right), 2007$ & No. of cases (and rate $\left.{ }^{a}\right), 2016$ \\
\hline All oral and pharynx sites & $35,076(10.89)$ & $44,419(11.7)$ \\
\hline Base of the tongue & $5661(1.72)$ & $8164(2.03)$ \\
\hline Soft palate and uvula ${ }^{b}$ & $870(0.27)$ & $743(0.19)$ \\
\hline Tonsil & $5791(1.76)$ & $8792(2.22)$ \\
\hline Oropharynx & $1507(0.46)$ & $2165(0.54)$ \\
\hline
\end{tabular}

HPV, human papillomavirus.

a Age adjusted per 100,000.

b The only HPV-associated sites with a decline in rate over this time period.

\section{TABLE 2}

\section{Number of HPV-associated and estimated number} of HPV-attributable cancer cases per year ${ }^{2}$

\begin{tabular}{c|l|l|l}
\hline Cancer site & $\begin{array}{l}\text { Average number of cancers } \\
\text { per year in sites where HPV is } \\
\text { often found (HPV-associated } \\
\text { cancers) }\end{array}$ & $\begin{array}{l}\text { Percentage probably } \\
\text { caused by any HPV } \\
\text { type }\end{array}$ & $\begin{array}{l}\text { Estimated number } \\
\text { probably caused by any } \\
\text { HPV type }\end{array}$ \\
\hline Cervix & 12,015 & $91 \%$ & 10,900 \\
\hline Vagina & 862 & $75 \%$ & 600 \\
\hline Vulva & 4009 & $69 \%$ & 2800 \\
\hline Penis & 1303 & $63 \%$ & 800 \\
\hline Anus & $6810^{\text {c }}$ & $91 \%$ & 6200 \\
Female & 4539 & $93 \%$ & 4200 \\
Male & 2270 & $89 \%$ & 2000 \\
\hline Oropharynx & 19,000 & $70 \%$ & 13,500 \\
Female & 3460 & $63 \%$ & 2200 \\
Male & 15,540 & $72 \%$ & 11,300 \\
\hline TOTAL & $\mathbf{4 3 , 9 9 9}$ & $\mathbf{7 9} \%$ & $\mathbf{3 4 , 8 0 0}$ \\
Female & $\mathbf{2 4 , 8 8 6}$ & $\mathbf{8 3 \%}$ & $\mathbf{2 0 , 7 0 0}$ \\
Male & $\mathbf{1 9 , 1 1 3}$ & $\mathbf{7 4 \%}$ & $\mathbf{1 4 , 1 0 0}$ \\
\hline
\end{tabular}

HPV, human papillomavirus.

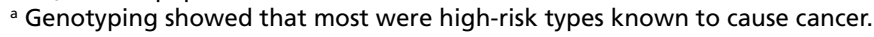

${ }^{b}$ Both anal and rectal squamous cell carcinomas.

${ }^{\mathrm{C}}$ Number is rounded.

1 or more doses of HPV vaccine, and only $51.1 \%$ were up to date. ${ }^{8}$ In contrast, $86.6 \%$ had received 1 or more doses of quadrivalent meningococcal vaccine; $88.9 \%$ had received 1 or more doses of tetanus, diphtheria \& acellular pertussis vaccine; $91.9 \%$ were up to date with 2 or more doses of measles, mumps \& rubella vaccine; and $92.1 \%$ were up to date with hepatitis B vaccine, with 3 or more doses. $^{8}$
Address parental concerns, including these 5 false beliefs

One study found 5 major false beliefs parents hold about HPV vaccine':

1. Vaccination is not effective at preventing cancer.

2. Pap smears are sufficient to prevent cervical cancer.

3. HPV vaccination is not safe.

4. HPV vaccination is not needed since 
TABLE 3

\section{Consider these USPSTF steps to reduce rates of oral, pharyngeal cancers ${ }^{3-6}$}

\begin{tabular}{l}
\hline Alcohol use screening \\
Screen for unhealthy alcohol use in primary care settings in \\
adults 18 years or older, including pregnant women, and \\
provide individuals engaged in risky or hazardous drinking \\
with brief behavioral counseling interventions to reduce \\
unhealthy alcohol use. (B recommendation) \\
\hline Tobacco use education and screening \\
\hline Provide education or brief counseling to prevent initiation of \\
tobacco use among school-aged children and adolescents. \\
(B recommendation) \\
\hline Ask all adults about tobacco use, advise them to stop using \\
tobacco, and provide behavioral interventions and US Food \\
and Drug Administration-approved pharmacotherapy for \\
cessation to adults who use tobacco. (A recommendation) \\
\hline Cervical cancer screening \\
\hline Screen for cervical cancer every 3 years with cervical cytology \\
alone in women ages 21 to 29 years. For women ages 30 to 65 \\
years, screen every 3 years with cervical cytology alone, every \\
5 years with high-risk human papillomavirus (hrHPV) testing \\
alone, or every 5 years with hrHPV testing in combination \\
with cytology (cotesting). (A recommendation) \\
\hline USPSTF, US Preventive Services Task Force.
\end{tabular}

TABLE 4

\section{ACIP recommendations for the use of HPV vaccine ${ }^{7}$}

\section{Individuals for whom vaccination is advised}

- HPV vaccine is recommended as routine vaccination for children ages 11 or 12 years. (Vaccination can start at age 9.)

- HPV vaccine is recommended for everyone through age 26 years, if not adequately vaccinated previously.

- Vaccination is not recommended for everyone older than age 26 years. However, adults ages 27 to 45 years may decide to get the HPV vaccine based on a discussion with their clinician, if they were not adequately vaccinated when they were younger.

\section{HPV dosing schedule}

- Two doses of HPV vaccine are recommended for most individuals starting the series before their 15th birthday.

- The second dose of HPV vaccine should be given 6-12 months after the first dose.

- Adolescents who receive 2 doses less than 5 months apart will require a third dose.

- Three doses of HPV vaccine are recommended for teens and young adults who start the series at ages 15 to 26 years, and for immunocompromised individuals.

- The recommended 3-dose schedule is 0, 1-2, and 6 months.

- Three doses are recommended for immunocompromised individuals (including those with HIV infection) ages 9 to 26 years.

ACIP, Advisory Committee on Immunization Practices; HIV, human immunodeficiency virus; HPV, human papillomavirus. most infections are naturally cleared by the immune system.

5 . Eleven to 12 years of age is too young to vaccinate.

There is some evidence that if clinicians actively engage with parents about these concerns and address them head on, same-day vaccination rates can improve. ${ }^{10}$

We can expect to see HPV-associated OPC decline in the coming years due to the delayed effects on cancer incidence by the HPV vaccine. These anticipated declines will be more dramatic if we can increase the uptake of the HPV vaccine.

References

1. Ellington TD, Henley SJ, Senkomago V, et al. Trends in the incidence of cancers of the oral cavity and pharynx-United States 2007-2016. MMWR Morb Mortal Wkly Rep. 2020;69:433-438.

2. CDC. HPV and cancer. 2019. www.cdc.gov/cancer/hpv/statistics/ cases.htm. Accessed June 29, 2020.

3. USPSTF. Unhealthy alcohol use in adolescents and adults: screening and behavioral counseling interventions. www. uspreventiveservicestaskforce.org/uspstf/recommendation/ unhealthy-alcohol-use-in-adolescents-and-adults-screening-andbehavioral-counseling-interventions. Accessed June 29, 2020.

4. USPSTF. Prevention and cessation of tobacco use in children and adolescents: primary care interventions. www. uspreventiveservicestaskforce.org/uspstf/recommendation/ tobacco-and-nicotine-use-prevention-in-children-andadolescents-primary-care-interventions. Accessed June 29, 2020.

5. USPSTF. Tobacco smoking cessation in adults, including pregnan women: behavioral and pharmacotherapy interventions. www. uspreventiveservicestaskforce.org/uspstf/recommendation/ tobacco-use-in-adults-and-pregnant-women-counseling-andinterventions. Accessed June 29, 2020.

6. USPSTF. Cervical cancer: screening. www.uspreventiveservices taskforce.org/uspstf/recommendation/cervical-cancer-screening. Accessed June 29, 2020.

7. CDC. Vaccines and preventable diseases. HPV vaccine recommendations. 2020. www.cdc.gov/vaccines/vpd/hpv/hcp/ recommendations.html. Accessed June 29, 2020.

8. Walker TY, Elam-Evans LD, Yankey D, et al. National, regional, state, and selected local area vaccination coverage among adolescents aged 13-17 years-United States, 2018. MMWR Morb Mortal Wkly Rep. 2019:68:718-723.

9. Bednarczyk RA. Addressing HPV vaccine myths: practical information for healthcare providers. Hum Vaccin Immunother 2019;15:1628-1638.

10. Shay LA, Baldwin AS, Betts AC, et al. Parent-provider communication of HPV vaccine hesitancy. Pediatrics 2018;141:e20172312. 